\title{
Rabaska
}

Revue d'ethnologie de l'Amérique française

\section{JARRY, LAURENT. Trésors de lames. Accordéons et bandonéons. Avant-propos de PIERRE ÉTAIX. Paris, Éditions Lelivredart, 2014, 304 p. ISBN 978-2- 35532-205-1}

\section{Steve Normandin}

Volume 17, 2019

URI : https://id.erudit.org/iderudit/1066031ar

DOI : https://doi.org/10.7202/1066031ar

Aller au sommaire du numéro

Éditeur(s)

Société québécoise d'ethnologie

ISSN

1703-7433 (imprimé)

1916-7350 (numérique)

Découvrir la revue

Citer ce compte rendu

Normandin, S. (2019). Compte rendu de [JARRY, LAURENT. Trésors de lames. Accordéons et bandonéons. Avant-propos de PIERRE ÉTAIX. Paris, Éditions Lelivredart, 2014, 304 p. ISBN 978-2- 35532-205-1]. Rabaska, 17, 307-311.

https://doi.org/10.7202/1066031ar d'utilisation que vous pouvez consulter en ligne.

https://apropos.erudit.org/fr/usagers/politique-dutilisation/ 
JARry, LaURent. Trésors de lames. Accordéons et bandonéons. Avant-propos de Pierre Étaix. Paris, Éditions Lelivredart, 2014, 304 p. ISBN 978-235532-205-1.

Le Français Laurent Jarry respire depuis plus de trente ans l'air agréablement musical que procure l'univers de l'accordéon. D'abord accordéoniste passionné de musique traditionnelle à danser, Jarry délaisse pendant près de trente ans la pratique de l'instrument pour plonger amoureusement au cœur de la conception et de la réparation des « boîtes à frissons » et de tout instrument à anches libres associé à la famille des accordéons (concertinas, bandonéons, systèmes chromatiques et diatoniques, etc.)

Son talent de réparateur et ses efforts de promotion lui confèrent une place à part chez les techniciens de l'accordéon en France : maître artisan en métier d'art, expert en accordéons et autres instruments à anches libres de l'Union française des experts spécialisés en antiquités et objets d'art (UFE). Sa réputation dépasse grandement les frontières hexagonales, lui assurant une fidèle clientèle parmi les virtuoses de tous horizons.

Ce carnet de bal semblerait bien rempli si on se laissait prendre au piège d'une danse panégyrique : Laurent Jarry chausse les souliers d'un grand collectionneur et la valeur historique de ses acquisitions, partenaires d'un siècle et demi d'évolution constante, donne le tournis. Inlassable tête chercheuse, il compile tout ce qui traite de la fabrication de l'instrument, passant du brevet au catalogue, des photos d'artistes aux méthodes d'enseignement, des enregistrements rares aux toiles de maître illustrant les soufflets dans toute leur splendeur.

Mais le facteur qu'il est sait admirer et bien expliquer le travail et la créativité dans la fabrication. Il lui faut sillonner les greniers poussiéreux, les ventes aux enchères et autres commerces en ligne pour dégoter les instruments anciens, rares, nécessaires à la nourriture de sa connaissance. Des accordéons exceptionnels sur lesquels se posent des touches de nacre, des parcelles de marqueterie et des kilos de celluloïd. Que d'essais et que d'erreurs dans ces prototypes de tous les systèmes et de tous les formats! Trop souvent, le collectionneur dit «normal», comme le danseur étoile qui s'approprie l'espace, garde cachées ses découvertes : Laurent Jarry prend l'heureux parti de partager la beauté et nous guide dans son musée intime... au cœur d'un opus qui pèse autant qu'un petit accordéon!

Trésors de lames paraît en octobre 2014 aux éditions Lelivredart. Cet ouvrage, quoique bien reçu du milieu musical mondial, et de l'univers des accordéonistes en particulier, demeure un ouvrage confidentiel malgré l'importance de l'œuvre réalisée. On ne peut que le regretter, tout en remerciant vivement Laurent Jarry d'avoir osé se lancer dans une telle entreprise. Trois 
cent quatre pages d'élégance visuelle, qui aurait pu simplement décliner l'histoire de l'accordéon sous nos yeux, en commençant par un des prototypes de l'autrichien Cyril Demian jusqu'aux dernières créations du Louisianais Marc Savoy, et nous tenir un discours technique professoral, purement historique et chirurgical, à l'intention d'un lectorat initié - ce qui est l'apanage d'une grande partie des livres consacrés à l'accordéon. Volontairement, Jarry tend la main au lecteur qui passe, peu importe son degré de connaissance musicale ou de l'univers entourant l'accordéon, en évitant les nomenclatures et les descriptions, les classifications rébarbatives. C'est un jeu de pistes pour les yeux : couleurs, détails, liens entre les instruments et les documents d'archives qui peuvent parfois s'y rattacher (photos personnelles d'anciens propriétaires musiciens, partitions musicales, mais aussi factures à l'achat de certains instruments).

Selon la formule consacrée et tout à fait à propos ici, rien n'est laissé au hasard. Dans la mise en forme, il y a du désordre ordonné : le concepteur se joue de nos sens et réussit à nous captiver. Cette surabondance ne peut être feuilletée à la sauvette tant les instruments illustrés sont différents : on se doit d'y revenir. Pourtant, même en un simple survol de quelques minutes, vous saisissez tout de suite la magie qui émane de l'instrument, de tous ces instruments réunis. Ce choix artistique, cette volonté d'aiguiser notre curiosité à l'envi, ce voyage dans le temps, c'est le tour de force réussi par l'auteur.

Avant de parler du choix cornélien de Laurent Jarry dans la présentation de sa collection personnelle d'accordéons venus du monde entier (sa collection dépasse largement les 500 pièces, accordéons mécaniques et automates compris), il me faut souligner le travail exceptionnel du photographe Olivier Eumont et la conception graphique de Cendrine Bonami-Redler. Pureté, légèreté, jeux d'ombres et mouvement dans l'immobilisme en sont les mots d'ordre. Le parcours sans faute nous est royalement présenté sur papier glacé. La mise en images permet une proximité, une intimité qu'aucun musée, aucune exposition ne pourra jamais offrir, à moins de posséder les instruments chez soi. Laurent Jarry a su, comme le réalisateur d'un film ou un grand chef cuisinier, s'entourer des meilleurs éléments pour rendre sa passion accessible. Les instruments chéris sont ainsi magnifiés et le rendu de chaque image se veut le juste et sincère hommage aux fabricants d'accordéons.

Dans son atelier de Montreuil, en banlieue sud de Paris, tout ce qui remue du soufflet et qui parle musique vient s'enquérir des services du technicien Laurent Jarry. Au hasard des rencontres et des projets, ce passionné a rencontré des gens pour qui l'accordéon avait une importance certaine ou un réel penchant pour la musique qui en émane. Ainsi, le clown de génie et réalisateur de films Pierre Étaix signe pour le livre un avant-propos qui codifie et fait la synthèse de tout ce que l'accordéon porte encore aujourd'hui dans son aura 
de légende : son accessibilité, sa force populaire, son impact auprès du public d'autrefois lorsque l'accordéon se faisait entendre dans les rues (Étaix, âgé de 85 ans au moment de livrer ses quelques lignes, évoque sa jeunesse), son évolution vers la musique dite « sérieuse ».

D'autres témoignages viennent émailler le parcours des images par leur évocation : presque tous les gens qui ont prêté leur concours et leur plume se servent du mot nostalgie - ces collaborateurs ne sont pas accordéonistes pour autant. Le geste nous paraît vibrant, malgré la redondance des propos tenus et la sensation d'un travail de commande. Certains tendent à la poésie, d'autres à l'analyse. Nous pouvons apprécier la tendresse envers l'accordéon à travers les témoignages des collaborateurs suivants : la pianiste et pédagogue d'origine roumaine Dana Ciocarlie, l'actrice Marie-Christine Barrault, le claveciniste Mario Raskin, le philosophe sociologue Gérard Rabinovitch, le compositeur Bernard Cavanna, le photographe et romancier Patrick Bard, le chroniqueur Francis Couvreux, le compositeur-pianiste Antoine Hervé et l'auteur-compositeur-interprète Francis Cabrel.

À mon avis, les plus beaux compléments de ce livre émanent de la recherche et du choix calibré de courts textes glissés en exergue par Laurent Jarry parmi les photographies. C'est autant de veines d'or sur un travail précieux. Extraits de chansons consacrées à l'accordéon (des grands succès aux plus obscurs refrains de la chanson française), traits de génie et d'humour d'auteurs célèbres, voire des critiques acerbes dans des revues ou des textes littéraires de la fin du XIX ${ }^{\mathrm{e}}$ siècle et, bien entendu, les éloges de l'instrument et de leurs virtuoses par le milieu littéraire (comment oublier l'amour réel que portait Francis Carco pour l'accordéon ?). Ainsi présentés, tous ces fragments de textes gagnent un éclat inattendu face à une judicieuse association d'idées. Le véritable respect porté à l'accordéon transparaît davantage dans ces tessons de citations.

La collection privée de Laurent Jarry foisonne de modèles somptueux. La facture d'accordéon est mondiale : à quelques exceptions près, les instruments présentés ici furent essentiellement conçus en Allemagne, en Italie, en France, en Belgique, en Russie, aux États-Unis et au Canada.

Accordéons primitifs aux touches sommaires, pour ne faire que des accords. Des prototypes de bandonéons avec des claviers piano au lieu des boutons traditionnellement vus sur ces appareils; accordéon aux coins de soufflet arrondis ; des instruments sur lesquels on a peint des personnages (l'ivrogne au pied d'un réverbère peint sur un bandonéon, magnifique œuvre d'art sur un instrument déjà remarquable), des décors (un accordéon italien représente Castelfidardo, village italien reconnu pour ses artisans d'accordéon, ou encore une scène de guerre avec des soldats portant la charge à coups de baïonnettes) ; accordéon aux aspects d'un appuie-livre ; accordéons dits 
« romantiques », avec leurs bascules comme première tentative de basses à la main gauche, des déesses grecques nues en nacre incrustée ; accordéon du fabricant Reisner et sa méthode signée de sa main (Louise Reisner, sa fille, fut une des premières virtuoses de l'instrument nouveau, donnant des concerts dans toute l'Europe); accordéon à pédales et colonne d'air venu d'Europe de l'est ; accordéon chromatique américain avec l'apparence de trois claviers de pianos superposés ; accordéons des années 1930 carrossés comme de vieilles Cadillac et enjolivés de pierreries et de traces fines gravées dans le celluloïd; accordéon de cirque surdimensionné, utilisé par des clowns musiciens et qui possède plus de 300 touches et ce, uniquement pour la main droite... Deux cent quatre-vingt-dix-sept accordéons particuliers, présentés dans ce livre, qui émergent d'une production mondiale titanesque étalée sur deux siècles.

Premier instrument de musique de l'ère industrielle (qui, comme son cousin l'harmonica, fut construit à la chaîne par des entreprises spécialisées), l'accordéon se distingue à la fois par l'apport commun de plusieurs artisans de nombreux pays européen et le succès de mise en marché d'un instrument grâce à une situation économique favorable à l'exportation. La migration de citoyens européens (notamment la communauté italienne et allemande) vers les deux Amériques a permis, dans des circonstances diverses et souvent matière à caution, l'implantation de diverses formes d'accordéons et de bandonéons.

Il y eut des usines d'accordéons et des petites fabriques (il y en a encore, heureusement !) mais sans l'apport direct de l'artisan, l'accordage des anches, l'ajustement et l'assemblage de toutes les pièces mécaniques qui composent chaque instrument seraient pratiquement impossibles. Tous ces détails apparaissant sur chacune des caisses d'instruments (sculptures, gravures, incrustations, toiles à l'intérieur des soufflets) ne sont que la partie visible de l'iceberg. Sans l'intervention du musicien, la magie des anches ne peut opérer. Le prestidigitateur Jarry connaît bien les entrailles de ces machines à leur contact quotidien. Pour lui, révéler l'esthétique de l'extérieur des accordéons lui semblait tout aussi important : le savoir-faire décoratif, le soin méticuleux de ces artisans de l'ombre qui habillaient les accordéons pour qu'ils accrochent le regard d'un côté flamboyant et spectaculaire ou porter le raffinement très loin par l'élégance des boiseries, de la nacre ou des gravures sur métaux.

Bien qu'il soit technicien en réparation, à moins de bris majeurs sur le plan visuel, jamais Laurent Jarry n'a voulu faire de retouche esthétique majeure, laissant la patine du temps exposer ses vérités. Il a désiré présenter les instruments « dans leur jus ", c'est-à-dire dans l'état de conservation dans lequel il les a retrouvés. Certains accordéons, particulièrement ceux construits au $\mathrm{XIX}^{\mathrm{e}}$ siècle, comprennent des pièces très fragiles, en ivoire ou en bois. Sachant 
la rareté de ces instruments produits en petit nombre, on ne peut qu'être béat d'admiration quant à leur découverte dans un si bon état de conservation. La simple présence de ces incunables au cœur de ce livre prend des allures de miracles. Il faut se pencher sur les clichés de ces spécimens pour comprendre à quel point sa cueillette s'impose comme une des plus importantes collections mondiales et pas seulement de l'accordéon, mais surtout de la facture instrumentale. Le travail de mise en page ici s'avère capital : il faut bénir le réalisme qui nous permet d'approcher les instruments de la collection Jarry.

En nous dévoilant sa caverne d'Ali-Baba remplie d'accordéons grâce à ce livre, Laurent Jarry concrétise une partie de ses rêves : en sa qualité d'artisan, il transmet à ses lecteurs son souci constant de préservation et son intérêt marqué pour le respect d'un patrimoine souvent méconnu et sous-estimé. Ce n'est pas qu'un simple outil de référence : ce livre révèle durablement et dans l'harmonie la beauté matérielle des accordéons.

Les soixante souscripteurs qui ont pu se procurer l'édition de luxe proposée par Laurent Jarry et ses éditeurs ont vu leur livre enchâssé dans un remarquable écrin de carton noir. Complément de programme : accompagnant la visite virtuelle des accordéons appartenant à Laurent Jarry, l'auteur et son éditeur ont eu l'excellente idée de reproduire en fac-similé l'intégralité d'un catalogue d'accordéons publié en Italie (date non mentionnée, sans doute au milieu des années 1920), également petit trésor de la collection Jarry. On y voit tous les modèles proposés par les usines Paolo Soprani \& Figli (compagnie dont la marque déposée existe toujours sous une autre entité). Des photos de la fabrique, les prix indiqués en lires, clichés d'instruments rares voisinant les accordéons populaires à bas prix. Ce cadeau « de collectionneur » porte dans ses pages illustrées une part du secret historique et de l'impact de la commercialisation de l'accordéon à l'échelle mondiale.

STEVe Normandin

Saint-Quay Perros, France

Labrecque, Simon. Un désir de liens, la mémoire qui nous agite. Montréal, Liber, 2019, 153 p. ISBN 978-2-89578-693-1.

Inclassable est le terme tout désigné pour qualifier à la fois l'auteur, tout comme son premier livre d'ailleurs, car tous deux sont grouillants de fébrilité. On sent chez lui l'urgence de dire, et dans l'essai, la prégnance de nommer des maux lancinants qui nous habitent en tant que groupe culturellement homogène jusqu'à ce jour. Simon Labrecque est jeune, né en 1985 sur la rive sud de Québec. Il est politologue de formation et fait probablement partie d'une classe d'auteurs à part qui voit plus vrai que grand. De sa plume agile, 